\title{
Benefit of Heart Rate Reduction in Heart Failure
}

\author{
Gerd Hasenfuss
}

Published online: 6 October 2010

(C) The Author(s) 2010. This article is published with open access at Springerlink.com

\section{Introduction}

Epidemiological studies have suggested that life expectancy is inversely related to resting heart rate and that the risks of development of heart failure and sudden cardiac death strongly increase with higher resting heart rates [1]. After myocardial infarction, the reduction in mortality with $\beta$-blocker treatment was correlated with the extent of heart rate reduction, and in patients with heart failure, a druginduced increase or decrease in heart rate has been associated with an increase or decrease in mortality, respectively [2,3]. The prognostic relevance of heart rate in patients with coronary artery disease and reduced ejection fraction recently has been demonstrated in the placebo arm of the prospective BEAUTIFUL (morBidity-mortality EvAlUaTion of the If inhibitor ivabradine in patients with coronary disease and left ventricULar dysfunction) study. The BEAUTIFUL study included patients with coronary artery disease and a left ventricular ejection fraction of less than $40 \%$. When patients with heart rates below and above 70 beats per minute were compared, cardiovascular death and heart failure hospitalization were significantly higher in the group of patients with higher heart rates [4].

\section{Pathophysiological Considerations}

$\beta$-Adrenoceptor blocking agents have proven to reduce mortality and to improve cardiac function in patients with

G. Hasenfuss $(\bowtie)$

Department of Cardiology and Pneumology,

University Medical Center,

Heart Research Center Göttingen, Robert-Koch-Strasse 40,

37075 Göttingen, Germany

e-mail: hasenfus@med.uni-goettingen.de heart failure and reduced ejection fraction. This beneficial effect of $\beta$-blockers may be partially related to heart rate reduction, but in addition, protection of the heart and other organs from catecholamines may contribute significantly to the effects of these drugs. How could a beneficial effect of selective heart rate reduction be explained? There are at least three major aspects to be considered. First, heart rate is linearly related with myocardial oxygen consumption [5]. Second, the decrease in heart rate prolongs duration of diastole, and thereby supports diastolic filling and coronary blood flow. Third, force-frequency relation is inverted in heart failure: while an increase in heart rate increases contractile performance in the nonfailing myocardium, higher heart rates are associated with a decline in contractile function in the failing myocardium. This has been shown in patient studies as well as in experimental work in isolated human myocardium [6,7]. Inverted forcefrequency relation was shown to result from disturbed calcium cycling at the level of the sarcoplasmic reticulum. Simply speaking, the higher the heart rate, the shorter the time available for diastolic calcium accumulation into the sarcoplasmic reticulum, which, because of disturbed sarcoplasmic reticulum calcium uptake, results in sarcoplasmic reticulum calcium depletion with higher heart rates [8].

SHIFT (Systolic Heart failure treatment with If inhibitor ivabradine Trial) investigates the effects of selective heart rate reduction with ivabradine in patients with heart failure [9].

\section{Study Design}

SHIFT is a randomized, double-blind, placebo-controlled, parallel-group trial including 6,558 patients who had 
symptomatic heart failure and a left ventricular ejection fraction below $35 \%$. Patients were randomly assigned to treatment with ivabradine (3,268 patients) or placebo (3,290 patients). All patients had to be in sinus rhythm with a heart rate of at least 70 beats per minute. The severity of the disease was assured by the inclusion criterion of heart failure hospitalization within the previous year. The patients were treated with ivabradine, $5 \mathrm{mg}$ twice daily, or matching placebo. After a period of 14 days, the ivabradine dose was increased to $7.5 \mathrm{mg}$ twice daily, unless the resting heart rate was below 60 beats per minute. If the heart rate was between 50 and 60 beats per minute, the dose was maintained at $5 \mathrm{mg}$ twice daily. If resting heart rate was lower than 50 beats per minute or the patient had signs or symptoms related to bradycardia, the dose was reduced to $2.5 \mathrm{mg}$ twice daily. At each follow-up visit, the ivabradine dose was adjusted. The primary end point was a composite of cardiovascular death or hospital admission for worsening heart failure. Secondary end points included heart failure mortality and hospitalization for heart failure. All of the events were analyzed on a time-to-first event basis. Changes in functional capacity were assessed by the New York Heart Association (NYHA) classification as well as by patient-reported and physician-reported global assessments.

\section{Results}

The median duration of follow-up was 22.9 months. Heart rate at inclusion was $79.9 \pm 9.6$ beats per minute and mean left ventricular ejection fraction was $29.0 \% \pm 5.1 \%$. At the beginning of the trial, mean ivabradine dosage was $6.4 \pm$ $1.6 \mathrm{mg}$ twice daily, which resulted in a heart rate reduction of $15.4 \pm 10.7$ beats per minute. Compared with pretreatment, when corrected for the change in the placebo group, the net heart rate reduction with ivabradine was 10.9 beats per minute. Cardiovascular death or hospital admission for worsening heart failure occurred in 937 (29\%) of the placebo group versus 793 (24\%) of the patients receiving ivabradine (hazard ratio $0.82 ; P<0.0001)$. This indicates that 26 patients would need treatment for 1 year to prevent one cardiovascular death or one hospital admission for heart failure. The effect was driven mainly by hospital admissions for worsening heart failure. From the secondary end points, death due to heart failure and hospital admission for worsening heart failure each was reduced by $26 \%$. Subgroup analysis suggests that the beneficial effect occurs predominately in patients with high heart rates. There was a small but significant improvement in the NYHA class. Serious adverse events were higher in the placebo than in the ivabradine group. Bradycardia leading to withdrawal from the study occurred in $1 \%$ of patients in the ivabradine group only.

\section{Discussion}

Ivabradine acts at the hyperpolarization-activated cyclic nucleotide-gated channel in the sinoatrial node, thus inhibiting the specific major pacemaker current ( $\mathrm{I}_{\mathrm{f}}$-funny current). Because this channel is selectively expressed in the sinus node, ivabradine does not influence atrioventricular conductance or cardiac performance. Thus, ivabradine is a selective heart rate-reducing agent. Therefore, SHIFT clearly shows that selective heart rate reduction in patients with heart failure and a heart rate above 70 beats per minute results in significant beneficial effects with respect to symptoms and prognosis. The patients have been pretreated according to international guidelines with the following characteristics: $90 \%$ of patients received angiotensin converting-enzyme inhibitors or angiotensin-receptor blockers, 90\% received $\beta$-blockers, and $84 \%$ received diuretics. It also is important to know that $25 \%$ of patients reached the target $\beta$-blocker dose and $57 \%$ of the patients reached more than $50 \%$ of the target dose. Thus, the patient cohort represents well-treated patients in the real world. Quite different from standards in the United States and Western Europe, the use of devices was rather low (cardiac resynchronization therapy: $1 \%$; implantable cardioverterdefibrillator: $4 \%$ ). This may relate to the fact that a significant number of patients had been included in countries with underuse of devices. It also is important to note that the average age of 60 years was rather young compared to a typical heart failure population.

\section{Conclusions}

SHIFT is an important new drug therapy trial that adds to the previous successful treatment strategies in patients with heart failure, including $\beta$-adrenoceptor blockers and renin-angiotensin-aldosterone system antagonists. Because adverse events of ivabradine treatment are rare and the beneficial effects of the drugs are impressive, we should consider treating patients with heart failure with a resting heart rate above 70 beats per minute with ivabradine in addition to guideline-recommended heart failure therapy. Of course, $\beta$-blocker treatment should be uptitrated to a maximum tolerable close, but in daily practise, because of hypotension and other side effects, a heart rate below 70 beats per minute is not frequently achieved with $\beta$-blocker treatment in patients with heart failure. SHIFT nicely fits with pathophysiological con- 
cepts indicating that high heart rate is harmful in patients with heart failure.

Disclosures Dr. Gerd Hassenfuss has received honoraria from Servier for giving talks on ivabradine.

Open Access This article is distributed under the terms of the Creative Commons Attribution Noncommercial License which permits any noncommercial use, distribution, and reproduction in any medium, provided the original author(s) and source are credited.

\section{References}

1. Cook S, Togni M, Schaub MC, et al. High heart rate: a cardiovascular risk factor? Eur Heart J. 2006;27:2387-93.

2. Kjekshus J. Heart rate reduction: a mechanism of benefit? Eur Heart J. 1987;8:115-22.
3. Swedberg K, Kjekshus J, Snapinn S. Long-term survival in severe heart failure in patients treated with enalapril. Ten year follow-up of CONSENSUS I. Eur Heart J. 1999;20:136-9.

4. Fox K, Ford I, Steg PG, et al. Heart rate as a prognostic risk factor in patients with coronary artery disease and left-ventricular systolic dysfunction (BEAUTIFUL): a subgroup analysis of a randomised controlled trial. Lancet. 2008;372:817-21.

5. Hasenfuss G, Holubarsch C, Heiss W, et al. Myocardial energetics in patients with dilated cardiomyopathy. Influence of nitroprusside and enoximone. Circulation. 1989;80:51-64.

6. Hasenfuss G, Holubarsch C, Hermann HP, et al. Influence of the force-frequency relationship on haemodynamics and left ventricular function in patients with non-failing hearts and in patients with dilated cardiomyopathy. Eur Heart J. 1994;15:164-70.

7. Mulieri LA, Hasenfuss G, Leavitt B, et al. Altered myocardial force-frequency relation in human heart failure. Circulation. 1992;85:1743-50.

8. Hasenfuss G, Pieske B. Calcium cycling in congestive heart failure. J Mol Cell Cardiol. 2002;34:951-69.

9. Swedberg K, Komajda M, Böhm M, et al. Ivabradine and outcomes in chronic heart failure (SHIFT): a randomised placebo-controlled study. Lancet. 2010;376:875-85. 